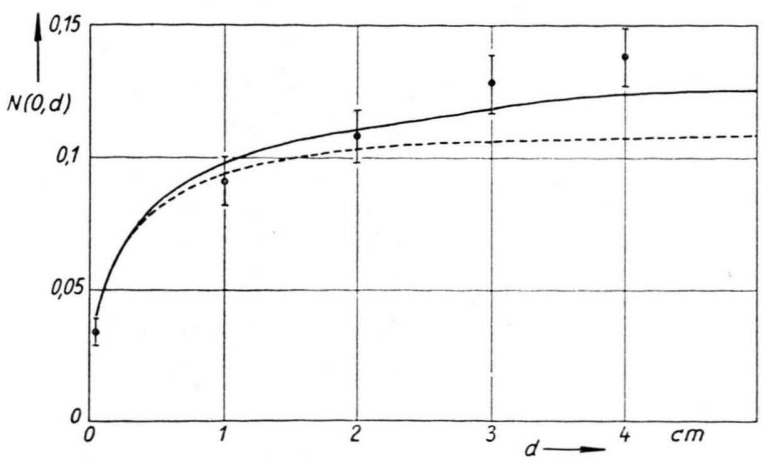

Abb. 9. Vergleich der gerechneten $N$-Wertekurve im Eisen mit experimentellen Ergebnissen ${ }^{11}$.

Kammer ein seitliches Weglaufen der die „zweite Generation“ erzeugenden $\gamma$-Quanten praktisch nicht vorkommt. Bei großen geometrischen Wegen der
$\gamma$-Quanten wird ein großer Teil der Stoßelektronen der zweiten Generation in einem so großen seitlichen Abstand vom $\mu$-Meson auftreten (vgl. Beginn von Abschn. III), daß eine eindeutige Zuordnung nicht mehr möglich ist, d. h. ein hoher Prozentsatz der "zweiten Generation" wird dann nicht gemessen. Wir verweisen für solche Fälle auf die in Abb. 1, 2 und 9 angegebenen Gesamtzahlen der direkt erzeugten Stoßelektronen.

Wir danken Herrn Prof. Heisenberg für förderndes Interesse und ebenso Herrn Prof. Wirtz, auf dessen Anregung diese Arbeit zustande kam.

Herrn Prof. Biermann möchten wir für die Erlaubnis zur Benutzung der Göttinger elektronischen Rechenmaschine G 1 danken, mit deren Hilfe numerische Auswertungen des vorliegenden Umfanges erst möglich wurden.

\title{
Ein Phosphormodell auf quantenmechanischer Grundlage
}

\author{
II. Übergangsmatrizen des Löschzentrums
}

\section{Von Harald Stumpf}

Aus dem Institut für theoretische und angewandte Physik der Technischen Hochschule Stuttgart

(Z. Naturforschg. 12 a, 465-478 [1957]; eingegangen am 18. März 1957)

\begin{abstract}
Das optische und elektrische Verhalten eines Phosphors, bei makroskopisch vorgegebenen $\mathrm{Zu}$ standsgrößen, wird durch sein Grundgitter und durch die Art und Anzahl der in ihm vorhandenen Störstellen bestimmt $(\S 10)$. An jeder Störstelle gehen kombinierte Elektron-Gitter-Prozesse vor sich, die quantenmechanisch durch Übergangsmatrizen beschrieben werden. Die Prozesse verlaufen entweder thermisch, d. h. unter Erhaltung der Gesamtenergie des Kristalls, oder elektromagnetisch, d. h. unter Emission oder Absorption von Photonen. An einem Löschzentrum überwiegen thermische Prozesse und führen zur strahlungslosen Rekombination angeregter Elektronen und Löcher. Hier werden die thermischen Übergangsmatrizen eines solchen Zentrums, z. B. einer $\mathrm{S}^{--}$-Lücke in $\mathrm{ZnS}$, für den Elektronen-Einfang und die Ionisation aus dem Grundzustand des Zentrums in das Leitungsund Valenzband berechnet. Die nichtadiabatischen Wechselwirkungsglieder des Kristalls bestimmen die Übergangsmatrizen. Zu ihrer Berechnung werden die Auswahlregeln für FrancK-Condon-Integrale bei beliebiger Nullpunktsverschiebung der Oszillatoren angegeben (\$ 11), unter der Annahme verschwindender Dispersion im longitudinalen optischen Zweig der Gitterschwingungen geeignete Eigenschwingungen abgeleitet (§ 12), der Grundzustand der Störstellenwellenfunktion des Elektrons (§ 13) und danach die Störstellenelektron-Gitter-Kopplung (§ 14) eingeführt. Daraus lassen sich die Nullpunktsverschiebungen der Gitter-Oszillatoren ableiten (§ 15). Die berechneten Matrizen folgen in § 16. Die optischen Übergangsmatrizen verschwinden in dieser Näherung vollständig. Diese Eigenschaften hängen wesentlich vom s-Funktionscharakter des Grundzustandes ab. Jedoch darf dies nicht als hinreichendes Kriterium für das Funktionieren eines Zentrums als Löschzentrum betrachtet werden, da noch weitere Größen, wie die energetische Höhe des Grundniveaus, Rekombinationen über angeregte Zustände, die Konzentration der Leitungsbandelektronen usw. das Gesamtverhalten bestimmen. Am Schluß werden in einer vergleichenden Tabelle die Annahmen anderer Autoren sowie bereits berechnete Fälle von Übergangsmatrizen einander gegenübergestellt.
\end{abstract}

\section{$\S$ 10. Einfachstes Phosphormodell ${ }^{1}$}

Wird ein Phosphor durch Absorption von Licht oder Energieaufnahme aus einer Teilchenstrahlung vom Grundzustand in einen angeregten Zustand ver-

1 § 1 bis 9 siehe H. Stumpf, Z. Naturforschg. 12 a, 153 [1957]; im folgenden mit I zitiert. setzt, so verläßt ein Bruchteil der Anregungsenergie den Kristall wieder als Fluoreszenz- und Phosphoreszenzstrahlung, während die übrige Energie in Wärme verwandelt wird. Zugleich mit der Energiespeicherung, die allmählich durch Phosphoreszenzstrahlung abgebaut wird, tritt in vielen Fällen lichtelektrische Leitung auf. Aus der Herstellungsweise 
eines Phosphors erkennt man, daß diese Prozesse mit der Existenz von Störzentren im Kristall verknüpft sind, und eine phänomenologische Analyse mit den Modellvorstellungen der Quantenmechanik lehrt, daß sich an diesen Störzentren kombinierte Elektron-Gitter-Anregungen abspielen müssen . Legt man diese Auffassung einem Phosphormodell zugrunde, so wird - abgesehen von den Valenzband-Leitungsband-Anregungen der Elektronen ${ }^{3}$ die Vielfalt der Phosphorreaktionen durch die möglichen Prozesse an einem Störzentrum, durch die verschiedenen Sorten von Störzentren, und deren Konzentrationen bestimmt. Betrachtet man zunächst einen Kristall mit einem Störzentrum, so lassen sich an diesem zwei Gruppen von Prozessen unterscheiden: elektromagnetische und thermische. Die ersten rühren von der Kopplung an das Strahlungsfeld, die zweiten von der Kopplung der Elektronen an das Gitter her. In jeder dieser Gruppen sind Elektronenübergänge

1. vom Valenzband in die Störstelle und umgekehrt,

2. vom Leitungsband in die Störstelle und umgekehrt,

3. innere Anregungen der Störstelle

möglich, wie Abb. 1 zeigt. Die Wahrscheinlichkeiten für diese Übergänge werden durch das Zentrum und seine Kopplung an die Umgebung (die gegebenenfalls auch die übrigen Störzentren enthält!) bedingt.

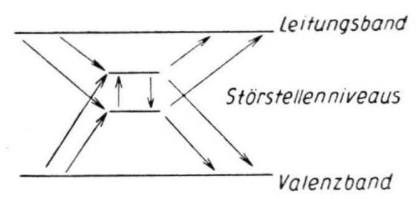

Berücksichtigt man die inneren Anregungen der Zentren nicht ${ }^{4}$, so muß ein Phosphormodell, wenn

2 Man vgl. hierzu etwa die zusammenfassenden Artikel von F. Stöckmann, Naturwiss. 39, 226 [1952] ; M. Schön, Techn. Wiss. Abh. der Osram 6, 49 [1953] ; F. A. KRöGER, Ergebn. exakt. Naturw. 29, 61 [1956] ; A. Rose, Phys. Rev. 97, 322 [1955]; W. Sсноттку u. F. Stöскмann, Halbleiterprobleme I, Verlag Vieweg, Braunschweig 1954, S. 81.

3 Band-Band-Rekombinationen werden ebenfalls ausgeschlossen. Sie sind im allgemeinen sehr unwahrscheinlich. Denn: nimmt man an, daß sich die Elektronen in Zuständen befinden, die durch B BocH-Funktionen beschrieben werden können, so ist die optische Übergangswahrscheinlichkeit, wenn man von der $\mathfrak{k}$-Auswahlregel absieht, zunächst in der Größenordnung der Übergangswahrscheinlichkeit freier Atome. Um die $\mathfrak{k}$-Auswahlregel mit Wahrscheinlichkeitsgewichten einzubeziehen, muß man beachten, daß ein in das Leitungsband angeregtes Elektron durch Streuprozesse, welche viel wahrscheinlicher sind als optische Prozesse, sofort den ursprünglichen $\mathrm{E}$-Wert der Anregung verliert, und nach - im Vergleich zur atomaren es die Ausbeuten und die Speicherung erklären will, die Konkurrenz strahlender und strahlungsloser Prozesse vorsehen. Das heißt, wir müssen beim theoretischen Ansatz auch im einfachsten Fall thermische Wechselwirkungen mit dem Gesamtkristall und elektromagnetische Wechselwirkungen mit dem Strahlungsfeld zulassen. Dies werden die nachfolgenden Betrachtungen erweisen. Da beide Prozeßgruppen an einer Störstelle zugleich auftreten, ist zunächst nicht offenkundig, daß sogar im einfachsten Modell zwei verschiedene Sorten von Störzentren notwendig sind. Wie wir aber einsehen werden, wird einerseits von der Präparation her die Aufteilung in zwei Störzentrensorten erzwungen, andererseits zeigt die nachfolgende theoretische Betrachtung mit Einschränkung, daß jede Übergangsart bevorzugt auf ein spezielles Strözentrum angewiesen ist. So ermöglichen die Aktivatoren fast nur strahlende Übergänge, wogegen an den Löschzentren strahlungslose Übergänge auftreten, die nur unter ungünstigen Bedingungen durch strahlende Übergänge verdrängt werden können.

Beschränkt man sich hierauf, so können an den zwei Störzentrensorten Phosphoreffekte studiert werden, obwohl erst das Hinzufügen weiterer Störzentren (traps, Koaktivatoren) eine direkte Korrespondenz des Modells zu den experimentellen Verhältnissen schafft.

Zur Demonstration der Behauptung über die gekoppelte Entstehung von Störstellen wählen wir den bekannten ZnS-Phosphor, der mit Kupfer aktiviert werde. Beim gesamten Aktivierungsvorgang muß die Neutralität des ursprünglichen Kristalls erhalten bleiben. Das wird gewährleistet, wenn bei allen Teilprozessen nur neutrale Teilchen in den Kristall ein-

Rekombinationszeit - kurzer Zeit gleichwahrscheinlich über die Energiefläche im $\mathfrak{k}$-Raum verteilt ist. Das gleiche gilt für das Loch. Daraus folgt, daß die atomare Übergangswahrscheinlichkeit noch jeweils mit der reziproken Anzahl von $\mathfrak{k}$-Werten auf der Energiefläche des Valenzund Leitungsbandes zu multiplizieren ist, um die BandBand-Rekombination zu erhalten. Der so entstehende Wert ist im allgemeinen bedeutend kleiner als die atomare Rekombinationswahrscheinlichkeit. Dies gilt natürlich nur für schwache Anregungen, und keine Exzitonen- und Polaronenzustände.

4 Das experimentell best untersuchte Beispiel dieser inneren Anregungen - das F-Zentrum - wurde von mehreren Autoren bereits ausgiebig theoretisch behandelt in bezug auf die strahlenden Übergänge. Andere Fragen, wie die Probleme der Anregung und temperatur-abhängigen Photoleitung, der Quantenausbeute, des Verhaltens in elektrischen Feldern usw., sollen in einer weiteren Arbeit behandelt werden. 
geführt oder herausgenommen werden. Dann ist folgender Kreisprozeß denkbar:

Zwei neutrale Kupferatome geben im Kristall ihr Außenelektron $\mathrm{ab}$ und werden $\mathrm{zu}$

$$
2 \mathrm{Cu} \rightarrow 2 \mathrm{Cu}^{+}+2 \mathrm{e}^{-} .
$$

Bei Substitution auf je einem Gitterplatz, an Stelle von $\mathrm{Zn}^{++}$, werden zwei $\mathrm{Zn}^{++}$frei. Nimmt man an, daß ein überschüssiges $\mathrm{Zn}^{++}$mit den zwei freien Elektronen rekombiniert

$$
\mathrm{Zn}^{++}+2 \mathrm{e}^{-} \rightarrow \mathrm{Zn}
$$

und aus dem Kristall wandert, sowie ein zweites herausgewandertes $\mathrm{Zn}^{++}$an der Oberfläche mit einem herausgewanderten $\mathrm{S}^{--}$zu einem zusätzlichen regulären Molekül wird, so ist die Neutralitätsbedingung gewahrt, obwohl schließlich im Innern eine $\mathrm{S}^{--}$-Lücke und zwei Aktivatoren übrig bleiben. Da sowohl bei der $\mathrm{S}^{--}$-Lücke als auch bei den Kupferatomen nicht alle Ladungen der Umgebung neutralisiert werden können, wirkt die Lücke auf weite Distanzen wie ein zweifach positiv geladenes und ein Aktivator wie ein einfach negativ geladenes Störzentrum. Mit dem Einbau von $2 \mathrm{Cu}$ ist also die Existenz einer Anionenlücke und damit eines weiteren Störzentrums erzwungen worden. Diese Lücke kann als Löschzentrum wirken, wogegen die Kupferstöratome wesentlich als Aktivatoren wirken.

Man sieht, daß das zweifach positiv geladene Zentrum ebensogut auch durch den Einbau des zweiten überschüssigen $\mathrm{Zn}^{++}$auf einem $\mathrm{Zwischengitterplatz}$ erzeugt werden kann. Unter dem zweifach positiv geladenen Zentrum kann daher zunächst entweder eine $\mathrm{S}^{--}$-Lücke oder ein $\mathrm{Zn}^{++}$auf einem $\mathrm{Zwischen-}$ gitterplatz verstanden werden. Soll die Störung jedoch als Löschzentrum wirken, so läßt sich eine Entscheidung treffen, welche von den zwei möglichen Konfigurationen diese Funktion übernehmen kann: Es ist jene, bei der ein angelagertes Störstellenelektron die geringere Bindung hat. Der Beweis wird später erbracht. Offensichtlich ist die Bindung des Störstellenelektrons an die Lücke geringer als an das doppelt geladene $\mathrm{Zn}^{++}$-Ion. Wir nehmen daher im folgenden nur die Existenz von Lücken an und betrachten den Fall von $\mathrm{Zn}^{++}$-Ionen auf $\mathrm{Z}$ wischengitterplätzen in unserem Modell als erheblich unwahrscheinlicher, so daß er auf die im Phosphor ablaufenden Prozesse keinen Einfluß hat. Eine strenge Entscheidung darüber, welcher Fall bevorzugt eintritt, kann nur durch eine Berechnung der Gitter- arbeiten für den Ein- und Ausbau gefällt werden. Diese Rechnungen führen wir hier nicht durch, da sie außerhalb der Theorie optischer und elektrischer Phosphorreaktionen liegen.

Der so aktivierte Kristall mit den dabei entstandenen Aktivatoren auf Gitterplätzen und den zweifach positiv geladenen Löschzentren wird dem Modell eines Kristallphosphors zugrunde gelegt, das wir nunmehr ausarbeiten wollen. Dazu müssen die Übergangswahrscheinlichkeiten für die verschiedenen Störstellenprozesse bestimmt und die Reaktionsgleichungen nach I abgeleitet werden. Dabei wird die dort erwähnte Wahl von quantenmechanischen statistischen Grundeinheiten notwendig, die als thermodynamisches Ensemble mit den Reaktionsgleichungen beschrieben werden. Diese Wahl hängt vom speziellen Modell ab und muß an ihm diskutiert werden. In unserem Fall haben wir einen Kristall vor uns, in dem eine gewisse Anzahl $2 \mathrm{~N}$ von Aktivatoren existiert, die wegen der Neutralitätsbedingung die Existenz der halben Anzahl $N$ von Lücken impliziert. Bei hinreichend geringer Konzentration der Defekte können wir diese als voneinander unabhängig ansehen, und unter der Annahme ihrer gleichmäßigen Verteilung den Kristall in ein System von Mikroblöcken zerlegen, in deren Mittelpunkt jeweils ein Störzentrum - Aktivator oder Löschzentrum sitzt, und deren räumliche Summe ohne Überschneidung das gesamte Kristallvolumen erfüllt. Es liegt nahe, einen solchen Mikroblock mit der Gesamtheit seiner Elektronen und Kerne zum Grundelement der thermodynamischen Statistik zu machen, so daß der Kristall als Ganzes ein Repräsentant eines Ensembles im Sinne der überlagerten thermodynamischen Statistik wird. Wegen der vorausgesetzten Unabhängigkeit der Mikroblöcke wird dabei freilich die räumliche Inhomogenität der Anregung, Energiekonzentration usw. im makroskopischen Sinne ausgeschlossen. Doch trifft dies erst ein sehr fortgeschrittenes Stadium der mikroskopischen Theorie, so daß wir zunächst bei der Untersuchung der einfachsten Möglichkeit, nämlich der Statistik der unabhängigen Mikroblöcke, verweilen.

Eine solche Statistik der Mikroblöcke kann nicht voraussetzungslos diskutiert werden. Vielmehr hängt die Diskussion von den Zielen der Theorie, den zugrunde liegenden theoretischen Vorstellungen und den benutzten Näherungen ab. Beim gegenwärtigen Stand kann es nicht die Frage sein, ob die Reaktionen eines bestimmten Phosphors numerisch genau 
wiedergegeben werden können, sondern es ist die Frage, ob eine konsequent vom Mikroskopischen her durchgeführte Theorie, unter den vorgegebenen Annahmen, überhaupt die charakteristischen Phosphorreaktionen liefert. Da alle Reaktionen und ihre Wahrscheinlichkeiten wesentlich von den Grundniveaus (ein Begriff, der in den weiteren Paragraphen noch erläutert wird) der Löschzentren und Aktivatoren abhängen, so führen wir diese als Parameter ein, um die ganze Schar von Lösungen in Abhängigkeit von diesen Parametern studieren zu können. Selbstverständlich lassen sich dann in speziellen Stoffen diese Niveaus theoretisch angeben. Die Beschränkung auf zwei Sorten von Störzentren mit zwei charakteristischen Niveaus soll weiterhin einen Beitrag zur Frage liefern, wieviel Störniveaus grundsätzlich notwendig sind, um - insbesondere in Hinblick auf die Photoleitung - eine vollständige Beschreibung des Phosphorverhaltens zu ermöglichen. Da wir der Ansicht sind, daß die Phosphorprozesse im allgemeinen über Nichtgleichgewichtszustände der Gitterschwingungen laufen, so befaßten wir uns in Teil I zunächst mit der Elektronen - Phononen-Reaktionskinetik. Dieser Teil war vom speziellen Modell unabhängig. Die folgenden Ausführungen in Teil II und III sind vom Modell abhängig und geben die Werte der Übergangsmatrizen und der daraus für I resultierenden Übergangswahrscheinlichkeiten. Auf diesem Gebiet hat bereits eine Reihe von Autoren den Versuch zur Berechnung unternommen. Die dabei durchgeführten Vereinfachungen sind im Anhang zu ersehen. Ein Vergleich zeigt, daß die explizit bekannten Daten nicht für unsere Untersuchungen hinreichen und die vorgenommenen Vereinfachungen unseren Absichten z. Tl. zuwiderlaufen.

Die hier angestellten Berechnungen gehen in zwei Stufen vor sich: in Teil II und III werden die Übergangsmatrizen unter der Voraussetzung nicht-polarisierbarer Elektronenhüllen der Ionen der Kristallumgebung abgeleitet. In Teil V wird diese Voraussetzung fallen gelassen und auch die Elektronenpolarisation quantenmechanisch berücksichtigt.
Bei unserer Polarisationsrechnung mit starren Ionen verwenden wir in Übereinstimmung mit den meisten anderen Autoren wegen der geringen Dispersion an Stelle des Frequenzspektrums der optischen Schwingungen nur eine einzige optische Frequenz. Dies bringt außerordentliche Vereinfachungen mit sich, die in früheren Arbeiten nicht benutzt wurden. Um konzentrationsabhängige Effekte zu studieren, wurde nicht der Grenzübergang des Mikroblockvolumens $V \rightarrow \infty$ vollzogen, sondern entsprechend unserer Statistik ein endliches von der Konzentration der Störstellen abhängiges $V$ beibehalten. Die weiteren Punkte kann man aus dem Anhang entnehmen.

Es bleibt noch die Abhängigkeit der Ergebnisse von der Störungsrechnung. Während alle vorgenannten Einschränkungen durch genauere Rechnungen aufgehoben werden können, ist die verwendete Störungsrechnung für die Übergangsmatrizen eine erste Näherung. Da in polaren Kristallen die Energiestufen aber weit auseinanderliegen, so sind die nichtadiabatischen Störglieder klein, und eine Störungsrechnung erster Ordnung in weitem Umfang wahrscheinlich gerechtfertigt. Sollte es sich trotzdem aus z. Zt. noch nicht angebbaren Gründen als notwendig erweisen, für die UUbergangsmatrizen höhere Näherungen zu verwenden, so wären die von Helmis aus der Quantenelektrodynamik übertragenen Verfahren vorzuziehen, obwohl die Schwierigkeiten ihrer expliziten Auswertung hier noch nicht bekannt sind.

Nach dieser Einführung können wir uns der Berechnung der Übergangsmatrizen am Löschzentrum zuwenden.

\section{$\S$ 11. Franck-Condon-Integrale}

Für die folgenden Erörterungen erweist sich der Übergang zu Normalkoordinaten in den Kernfreiheitsgraden als zweckmäßig. Die Übergangsmatrizen für die kristalleigenen Störglieder (I, 3) lauten dann mit einem bestimmten Normalkoordinatensatz

$$
q_{\varrho}^{n}(n=\text { const, } \varrho=1, \ldots, N)
$$

$$
\int \psi_{j}^{*} \varphi_{!}^{j *}\left(-\frac{\hbar^{2}}{M} \sum_{\varrho=1}^{N} \frac{\partial}{\partial q_{\varrho}^{n}} \psi_{n} \frac{\partial}{\partial q_{\varrho}^{n}} \varphi_{m}^{n}-\frac{\hbar^{2}}{2 M} \sum_{\varrho=1}^{N} \varphi_{m}^{n} \frac{\partial^{2}}{\partial q_{\varrho}^{n 2}} \psi_{n}\right) \mathrm{d} \tau,
$$

wobei die elektronischen Funktionen $\psi_{j}{ }^{*}$ bzw. $\psi_{n}$ wegen der adiabatischen Näherung die Kernkoordinaten als Parameter enthalten. Entwickelt man $\psi_{j}$ nach den zugehörigen $q_{\varrho}^{j}$, und $\psi_{n}$ nach den zugehörigen $q_{\varrho}^{n}$, so kann man die Integration über Elektronen und Kernfreiheitsgrade separieren und erhält 
für den Kernanteil Integrale der Art

$$
\int \varphi_{l}^{j *} \prod_{i}\left(q_{i}^{j}\right)^{\alpha_{i}^{j}} \prod_{h}\left(q_{h}^{n}\right)^{\alpha_{h}^{n}} \varphi_{m}^{n} \mathrm{~d} q_{1}^{n} \ldots \mathrm{d} q_{N}^{n}
$$

und

$$
\int \varphi_{l}^{j *} \prod_{i}\left(q_{i}^{j}\right)^{\alpha_{i}^{j}} \prod_{h}\left(q_{h}^{n}\right)^{\alpha_{h}^{n}} \frac{\partial}{\partial q_{\varrho}^{n}} \varphi_{m}^{n} \mathrm{~d} q_{1}^{n} \ldots \mathrm{d} q_{N}^{n}
$$

wenn der elektronische Anteil nicht angeschrieben wird. Die $\alpha_{i}^{j}$ bzw. $\alpha_{k}^{n}$ sind dann die Potenzzahlen der einzelnen Glieder der TAYLoR-Entwicklung. Die Integrale erster Art (4) treten auch in den optischen Übergangselementen auf, so daß wir mit der Diskussion von (4) und (5) alle möglichen Integralkombinationen erfassen. Weiterhin brauchen wir nur (4) zu untersuchen. Mit Hilfe der Beziehung

$$
\frac{\partial \Phi_{n}}{\partial \xi}=-\left(\frac{n+1}{2}\right)^{1 / 2} \Phi_{n+1}+\left(\frac{n}{2}\right)^{1 / 2} \Phi_{n-1},
$$

welche für Oszillatorenfunktionen

$$
\Phi_{n}=C_{n} H_{n}(\xi) e^{-\xi^{2} / 2}
$$

gilt, wobei $H_{n}(\xi)$ das Hermitesche Polynom $n$-ter Ordnung sei, läßt sich nämlich für eine einzelne Oszillatorfunktion, bei Einführung der von uns benutzten Normalkoordinaten durch

$$
\xi=q\left(\frac{\omega M}{2 \hbar}\right)^{1 / 2}
$$

ableiten

$\frac{\partial \Phi_{n}}{\partial q_{j}^{n}}=-\left(\frac{n+1}{2}\right)^{1 / 2}\left(\frac{\omega M}{2 \hbar}\right)^{1 / 2} \Phi_{n+1}+\left(\frac{n}{2}\right)^{1 / 2}\left(\frac{\omega M}{2 \hbar}\right)^{1 / 2} \Phi_{n-1}$.

Ersetzt man nun $\varphi_{m}^{n}$ durch

$$
\varphi_{m}^{n} \equiv \Phi_{1}^{n}\left(q_{1}^{n}\right) \ldots \Phi_{N}^{n}\left(q_{N}^{n}\right)
$$

und beachtet die Formel (9), so läßt sich (5) auf (4) reduzieren. Die genaue Indexvertauschung führen wir in $\S 16$ aus.

Bei Einsetzen von (10) in (4) und der analogen Formel für $\varphi_{l}^{j}$ erhält man $s o$

$$
\int \Phi_{1}^{j}\left(q_{1}^{j}\right)^{*} \ldots \Phi_{N}^{j}\left(q_{N}^{j}\right)^{*} \prod_{i}\left(q_{i}^{j}\right)^{\alpha_{i}^{j}} \prod_{h}\left(q_{h}^{n}\right)^{\alpha_{h}^{n}} \Phi_{1}^{n}\left(q_{1}^{n}\right) \ldots \Phi_{N}^{n}\left(q_{N}^{n}\right) \mathrm{d} q_{1}^{n} \ldots \mathrm{d} q_{N}^{n}
$$

jedoch treten in den Integralen noch verschiedene Integrationsvariablen $q_{\varrho}^{j}$ und $q_{\varrho}^{n}$ auf. Bei unserer Definition der Normalkoordinaten sind die $q_{e}^{j}$ und die $q_{\varrho}^{n}$ durch eine unitäre Transformation und eine Nullpunktsverschiebung verbunden ${ }^{4 a}$. Es gilt also

$$
q_{\mu}^{j}=\sum_{\varrho} U_{\mu \varrho}(j, n) q_{\varrho}^{n}+a_{\mu} .
$$

Nimmt man an, daß die Eigenschwingungen im Verlauf der Prozesse ihre Gestalt nicht wesentlich ändern (vgl. § 12), so geht $U_{\mu \rho}$ in eine Einheitsmatrix über, und man erhält

$$
q_{\mu}^{j}=q_{\mu}^{n}+a_{\mu} .
$$

Da das zugehörige Potential der quantenmechanischen Gittergleichungen nicht durch die gleiche unitäre Transformation bestimmt werden kann, so sind Änderungen der Eigenfrequenzen neben den Nullpunktsverschiebungen noch möglich. Wir werden in den kommenden Paragraphen einsehen, daß im wesentlichen nur Transformationen der Art (13) auftreten. Einsetzen von (13) in (11) gestattet eine weitere Separation des Integrals, so daß man für einen separierten Anteil das Integral ${ }^{5}$

$$
\int \Phi_{l_{i}^{j}}^{j_{i}}\left(q_{i}^{n}+a_{i}\right)^{*}\left[q_{i}^{n}+a_{i}\right]^{\alpha_{i}^{j}}\left[q_{i}^{n}\right]^{\alpha_{i}^{n}} \Phi_{l_{i}^{n}}^{n}\left(q_{i}^{n}\right) \mathrm{d} q_{i}^{n}
$$

erhält, und ganz analog die übrigen Anteile. Für $\alpha_{i}^{j}$ und $\alpha_{i}^{n}=0$ erhält man

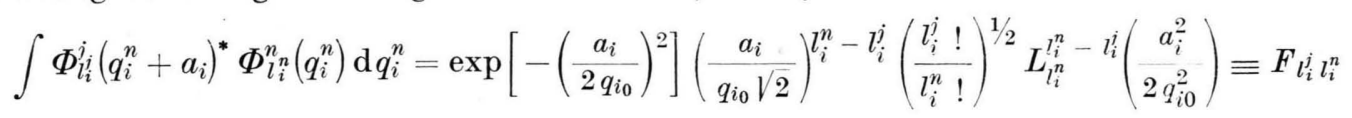

unter der Voraussetzung, daß die beiden Oszillatoren gleiche Frequenzen aufweisen. Dabei ist $L_{n}^{m-n}$ ein Laguerresches Polynom ${ }^{6}$ und $q_{i 0}=\left(\hbar / M \omega_{i}\right)^{1 / 2}$.

4a s. h. H. Stumpf, Z. Naturforschg. 10 a, 971 [1955], § 10.

$5 \mathrm{Im}$ folgenden verwenden wir die Indizierung nach I, § 4.
Die Formel gilt für $i_{i}^{n} \geqq \dot{l}_{i}^{j}$. Für $i_{i}^{n} \leqq l_{i}^{j}$ ist darin $l_{i}^{n}$ und $l_{i}^{i}$ zu vertauschen, und außerdem $a_{i}$ durch $-a_{i}$ zu ersetzen.

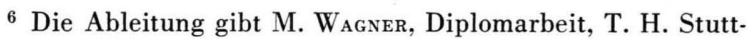
gart 1957.
} 
In den folgenden Rechnungen werden wir nur Formeln des Typus (15) verwenden. Dies ist die sogenannte Condon-Näherung. Sie bedeutet nicht, daß die elektronischen Wellenfunktionen gänzlich $q$-unabhängig sind. Vielmehr müssen ja $q$-Ableitungen gebildet werden. Aber (15) sagt aus, daß nach Bildung dieser $q$-Ableitungen im elektronischen Anteil eine Reihenentwicklung vorgenommen und von dieser nur der $q$-unabhängige Teil verwendet wird, so daß im Effekt in $\psi q$-Abhängigkeiten bis zu quadratischen Gliedern (Näherung harmonischer Oszillatoren) berücksichtigt werden. Das gilt allerdings nur für die Kristall-Störglieder (3). Die optischen Dipolmomente werden in dieser Näherung tatsächlich $q$-unabhängig, was Anregungen der Kernschwingungen aber nicht ausschließt, da diese bereits in den FrancK - Condon-Integralen (15) auftreten. Die mit $q$ behafteten Glieder sind nur für die feinere Struktur der Absorptions- und Emissionsbanden verantwortlich, die wir hier nicht weiter studieren werden.

\section{§ 12. Eigenschwingungen und Verschiebungen bei rein elektrostatischen Störkräften}

Für die theoretische Beschreibung der Phosphorreaktionen ist eine Kenntnis der Eigenschwingungen des Kristalls, einschließlich der lokalisierten Schwingungen der Störstelle und ihrer Umgebung notwendig, da die Ionenverschiebungen beim Elektronenübergang in die Störstelle nach den zugehörigen Kernschwingungsfunktionen entwickelt werden müssen. Im allgemeinen Fall bedarf es aller Eigenschwingungen des Kristalls, um die Kernverschiebungen exakt darzustellen. Aber man kann die notwendige Anzahl allein durch Symmetrieüberlegungen beträchtlich reduzieren. So werden bei der Besetzung eines s-Zustandes des Störatoms auf einem Zwischengitterplatz nur radiale Eigenschwingungen der umgebenden Ionen angeregt. Der einfachste Fall jedoch liegt vor, wenn überhaupt nur eine einzige Eigenschwingung angeregt wird. Wir zeigen, daß dies tatsächlich eintritt unter Voraussetzungen, die vom Charakter der Störstelle abhängig sind. Im Grenzfall kann man nämlich zwei Störstellentypen zufolge ihrer verschiedenartigen Kopplung an die Umgebung unterscheiden: jene, die die Definition eines wohlabgegrenzten Störmoleküls, mit von der Kristallumgebung stark verschiedenen inneren Bindungskräften gestatten, und andere, deren Bindungskräfte an die Kristallumgebung sich nur wenig von denen des ungestörten Kristalls unterscheiden, wobei aber die schwache Störung sich weit in den Kristall erstreckt. Zu den ersten wären elektrisch neutrale Störungen zu rechnen, die in dichten Gittern zu einer starken Deformation der inneren Elektronenhüllen und damit zu starken Abstoßungskräften führen, zur zweiten Art aber elektrisch geladene Störstellen in weiten Gittern, bei denen die elektrostatischen Fernkräfte überwiegen, und die quantenmechanische Verquetschung der Elektronenhüllen nur von geringem Einfluß ist. Für die erste Art ist die Frequenz und die Gestalt der Eigenschwingungen in Abhängigkeit vom Störmolekül bekannt. Dieser Fall ist bei Phosphorstörstellen jedoch nicht realisiert. Vielmehr treten dort Störstellen vom zweiten Typ auf. Für diese leiten wir nun Eigenschwingungen $a b$, und zeigen, daß diese so gewählt werden können, daß ein Eigenvektor in erster Näherung mit dem Ionenverschiebungsvektor übereinstimmt.

Dazu betrachten wir die klassischen dynamischen Gittergleichungen des gestörten Kristalls

$$
\sum_{k} H_{i k} \xi_{k}^{\mu}=\omega^{\mu 2} M \xi_{i}^{\mu} .
$$

$H_{i k}$ ist der Gitteroperator des gestörten Systems und $\xi_{k}^{\mu}=\left(X_{k}^{\mu}-X_{k}^{0}\right)(k=1, \ldots, N)$ der $\mu$-te Eigenvektor des Systems. Ferner werden ungefähr gleiche Ionenmassen vorausgesetzt $M_{i} \approx M_{k}=M$. Der vollständige Satz von Eigenvektoren $\xi_{k}^{\mu}$ erfüllt aber nicht nur die Gittergleichungen, sondern befriedigt auch die Symmetriegruppe des gestörten Gitters mit den Operationen $O_{\mathrm{S}}$. Für jene Eigenvektoren $\mu_{\varkappa}(\varkappa=1, \ldots)$, die einer eindimensionalen, nicht auf Hauptachsen bezogenen Darstellung der Gruppe zugeordnet sind, gilt daher die Eigengleichung

$$
\sum_{k} O_{\mathrm{S}_{i k}} \xi_{k}^{\mu_{\varkappa}}=o_{\mathrm{s}}^{\mu_{\varkappa}} \xi_{i}^{\mu_{\varkappa}}
$$

wenn $O_{\mathrm{S}_{i k}}$ die Darstellungsmatrizen der Gruppe sind. Da für den vorausgesetzten Störungstyp nur eine kleine Änderung der Bindungskräfte eintreten soll, die wir durch einen Parameter $\lambda$ kennzeichnen, mit $\lambda \ll 1$, so läßt sich der Gitteroperator anschreiben als

$$
H_{i k}=H_{i k}^{0}+\lambda H_{i k}^{1}
$$

und die Lösung in Form einer Potenzreihe nach $\lambda$

$$
\xi_{k}^{\mu}=\xi_{k}^{0 \mu}+\lambda \xi_{k}^{1 \mu}+\ldots
$$

Für einen Eigenvektor, der der eindimensionalen 
Darstellung angehört, muß neben (16) auch (17) erfüllt sein, was für unsere Entwicklung liefert

$$
\sum_{k} H_{i k}^{0} \xi_{k}^{0 \mu_{\varkappa}}=\omega^{02} M \xi_{i}^{0 \mu_{\varkappa}}
$$

und

$$
\sum_{k} O_{\mathrm{S}_{i k}} \xi_{k}^{0 \mu_{\varkappa}}=o_{\mathrm{s}}^{\mu_{\varkappa}} \xi_{k}^{0 \mu_{\varkappa}},
$$

da der Symmetrieoperator von $\lambda$ unabhängig ist. Die Gleichungen für höhere Ordnungen schreiben wir hier nicht an. (20) und (21) geben die Forderungen an die Lösungen in nullter Näherung wieder und bestimmen diese eindeutig.

Weitere Aussagen lassen sich über die Eigenvektoren nullter Näherung und die zugeordneten Frequenzen machen, wenn das Gitter weitgehend dem klassischen Polarisationsgesetz gehorcht. Dies ist für polare Gitter bei den optischen Schwingungen der Fall. Da hier nur Verschiebungen der Ionen in den Zellen stattfinden, ist die Polarisation nicht als Mittelwert von deformierten Raumbereichen, sondern von Ionenverschiebungen innerhalb der Zellen definiert. Dann ergibt aber bereits jede Zelle ein Dipolmoment, und die makroskopische Definition der Polarisation kann bis auf Gebiete von Zellengröße erstreckt werden, d. h. aber, dieser Polarisationsausdruck wird eine gute atomistische Annäherung bilden.

Um also die Frequenzen und Eigenvektoren abzuleiten, betrachten wir die statischen Gittergleichungen

$$
\sum_{k} H_{i k}^{0} \xi_{k}=K_{i} .
$$

In ihnen seien die Kräfte $K_{i}$ elektrostatischen Ursprungs, und mögen von einer Ladungsverteilung herrühren, welche der Symmetriegruppe $O_{\mathrm{S}}$ als eindimensionale Darstellung genügt. Dann gehen die Gln. (22) über in

$$
\sum_{k} H_{i k}^{0} \xi_{k}=\mathfrak{D}\left(\mathrm{r}_{\mathrm{i}}\right) e_{\mathrm{i}},
$$

wobei $\mathfrak{D}\left(\mathrm{r}_{\mathrm{i}}\right)$ das von jener Ladungsverteilung herrührende Feld am $i$-ten Ion sei ${ }^{7}$. Da das elektrostatische Feld die entgegengesetzt geladenen Ionen in entgegengesetzter Richtung verschiebt, so kann man diese Verschiebung eine „optische“ Verschiebung nennen; bei ihr werden zusätzliche Dipolmomente in jeder Zelle erzeugt. Für die statische

\footnotetext{
7 in den Gittergleichungen (23). Denn diese sind atomistisch und enthalten bei Einführung der Ladung in das ungestörte Gitter die Superposition aller nichtabgeschirmten
}

Gleichung kann man deshalb mit Hilfe der makroskopischen Elektrostatik weitere Aussagen machen. Nachdem die Verschiebungen gemäß (23) eingetreten sind, wirkt im neuen Gleichgewichtszustand die Stör-Ladung mit dem elektrischen Feld $\mathcal{E}=\mathfrak{D} / \varepsilon$ auf das Gitter. Die Gitterreaktion ist ein Feld $\mathfrak{P}$, die Polarisation, welche in jedem Punkt proportional zu EF ist.

$$
\mathfrak{P}=\frac{\varepsilon-1}{4 \pi} \mathfrak{F}=\frac{\varepsilon-1}{4 \pi \varepsilon} \mathfrak{D} .
$$

Da die Polarisation mit den Ionenverschiebungen verknüpft ist, gilt für ein Volumelement

$$
\sum_{i \text { in } \tau_{j}} e_{i} \xi_{i}=\mathfrak{\Re} \tau_{j}=\frac{\varepsilon-1}{4 \pi \varepsilon} \mathfrak{D} \tau_{j} .
$$

Bei optischen Verschiebungen läßt sich die Polarisation noch in den Gitterelementarzellen definieren, und man kann speziell als Volumelement eine Zelle des Dipolmediums $\tau_{j}$ wählen, in der jeweils ein Ion enthalten ist, wobei man erhält

$$
e_{j} \xi_{j}=\frac{\varepsilon-1}{4 \pi \varepsilon} \mathfrak{D}\left(\mathrm{r}_{j}\right) \tau_{j} .
$$

Die Umkehrung dieses Zusammenhanges liefert

$$
\mathfrak{D}\left(\mathrm{r}_{j}\right)=\frac{4 \pi \varepsilon}{(\varepsilon-1)} \frac{1}{\tau_{j}} \xi_{j} e_{j}
$$

und mit (23) verkoppelt

$$
\sum_{k} H_{i k}^{0} \xi_{k}=\frac{4 \pi \varepsilon}{(\varepsilon-1)} \frac{e^{2}}{\tau_{i}} \xi_{i} .
$$

Andererseits sollen die dynamischen Gln. (20) und (21) gelten. Mit

und

$$
\begin{aligned}
\omega & =\sqrt{\frac{4 \pi \varepsilon}{(\varepsilon-1)} \frac{e^{2}}{\tau M}} \\
\xi_{i}^{\mu} & =\frac{1}{\gamma^{\mu}} \mathfrak{D}\left(\mathfrak{r}_{i}\right) e_{i}
\end{aligned}
$$

ist das erfüllt, wenn $\gamma^{u}$ die Normierungskonstante des Eigenvektors bedeutet.

Wie man sieht, können als Eigenvektoren alle jene Felder auftreten, die eine zu $O_{\mathrm{S}}$ gruppensymmetrische Ladungsverteilung aufweisen, und als Eigenfrequenz tritt nur eine einzige Frequenz (29) auf. Die klassische Näherung liefert also den Fall verschwindender Dispersion; eine Näherung, die häufig benutzt wird, und die, wie man sich durch

elektrostatischen Kräfte. Erst nach eingetretener Verschiebung der Umgebung superponieren sich sämtliche Felder, so daß eine Abschirmung der eingeführten Ladung entsteht. 
Betrachtung des Frequenzganges optischer Schwingungen überzeugt, auch durch die wirklichen Verhältnisse weitgehend gerechtfertigt wird ${ }^{8}$. Wegen der einen Frequenz (29) sind alle Eigenvektoren entartet. Bei entarteten Eigenvektoren ist aber mit $\xi_{k}^{\mu}$ auch eine beliebige Kombination $\sum_{\mu} c_{\mu} \xi_{k}^{\mu}$ ein Eigenvektor, und diese Kombinationen liefern gerade die Mannigfaltigkeit der möglichen Eigenvektoren (30) mit gruppensymmetrischer Ladungsverteilung.

Diesen Umstand machen wir uns zunutze. Indem wir beim Fall verschwindender Dispersion bleiben, läßt sich nach (30) eine Eigenschwingung $\xi_{i}{ }^{1}$ superponieren von der Gestalt

$$
\xi_{i}^{1}=\left(\gamma^{1}\right)^{-1} \int \frac{\psi^{*}\left(\mathrm{r}^{\prime}\right) \psi\left(\mathrm{r}^{\prime}\right)}{\left|\mathrm{r}_{\mathrm{i}}-\mathrm{r}^{\prime}\right|^{2}} \mathrm{~d} \tau^{\prime} e_{i} e,
$$

wenn $\psi(\mathrm{r})$ die Grundwellenfunktion eines Elektrons am Löschzentrum darstellt und die $\xi_{i}{ }^{1}$ als Radialkomponenten der Ionenverschiebungen in bezug auf ein mit der Störung verknüpftes Polarkoordinatensystem angesehen werden. Da (31) zugleich auch proportional der elektrostatischen Verschiebung der Ionen beim Einfang eines Elektrons in die Störstelle ist, so gibt es eine Eigenschwingung, welche mit dieser Verschiebung identisch ist. Von dieser Relation werden wir im folgenden Gebrauch machen. Um die anderen Eigenschwingungen kümmern wir uns in dieser Näherung nicht, da sie aus der weiteren Rechnung herausfallen werden.

\section{§ 13. Die Grundkonfiguration}

Da die Übergangsmatrixelemente (3) und (I, 8) von den parameterabhängigen Wellenfunktionen der Elektronen mitbestimmt werden, so müssen wir diese Funktionen im folgenden berechnen. In der adiabatischen Näherung erhält man dafür die Elektronengleichung

$\left[-\frac{\hbar^{2}}{2 m} \sum_{i=1}^{N^{\prime}} \frac{\partial^{2}}{\partial x_{i}^{2}}+V\left(x_{i} X_{k}\right)\right] \psi\left(x_{i} X_{k}\right)$

$$
=U\left(X_{k}\right) \psi\left(x_{i} X_{k}\right) .
$$

In ihr sind neben den Leitungselektronen noch die Valenzelektronen enthalten, d. h. alle jene Elektronen, die an Prozessen im optischen Bereich teilnehmen können. Die strenge Lösung dieser Gleichung ist für beliebige Parameterwerte $X_{k}$ nicht möglich.

8 Vgl. Th. D. Schultz, Techn. Report No. 9, Massachusetts Institute of Technology 1956, S. 9.
Jedoch bietet sich sogleich aus der Modellvorstellung ein Reduktionsverfahren an: Der Realkristall wird aus der Störstelle und der übrigen Kristallumgebung aufgebaut gedacht. Dementsprechend wird man auch die zugehörigen Elektronenwellenfunktionen aus zwei Anteilen zusammensetzen: der Störstellenwellenfunktion, deren Elektronenkoordinaten wir mit $x$ bezeichnen, und die Wellenfunktion der Umgebung mit den Elektronenkoordinaten $x_{i}$. In der Theorie der starren Ionen hängt die Umgebungswellenfunktion nicht vom elektronischen Zustand der Störstelle ab, und wir können als Wellenfunktion ansetzen

$$
\psi\left(x_{i} X_{k}\right)=\psi_{\mathrm{S}}\left(x X_{k}\right) \psi_{n}\left(x_{i} X_{k}\right) .
$$

Setzt man dies in Gl. (32) ein, multipliziert von links mit $\psi_{n}{ }^{*}$ und integriert über $x_{i}$, so ergibt sich eine Gleichung für $\psi_{\mathrm{S}}\left(x, X_{k}\right)$

$\left[-\frac{\hbar^{2}}{2 m} \sum \frac{\partial^{2}}{\partial x^{2}}+V\left(x, X_{k}\right)+V\left(X_{k}\right)\right] \psi_{\mathrm{S}}=U\left(X_{k}\right) \psi_{\mathrm{S}}$.

Dabei ist $V\left(x, X_{k}\right)$ das Wechselwirkungsglied des Störstellenelektrons mit der Gitterumgebung, und $V\left(X_{k}\right)$ die Gitterenergie, welche nicht nur die Energie der Kristallumgebung, sondern auch die Wechselwirkung der „nackten“ Störstelle mit der Gitterumgebung enthält. Dieser Anteil wirkt nicht auf die Störstellenwellenfunktion. Dagegen der erste. Ihn zerlegen wir in zwei Teile: ein Teil $V^{(1)}\left(x, X_{k}^{0}\right)$, der dem Zustand entspricht, in dem sich das Gitter bei besetzter Störstelle befindet, wobei $X_{k}^{0}$ die Ruhelagen des Gitters kennzeichnen, und einem zweiten Anteil, der die zusätzliche Wechselwirkungsenergie $V^{(2)}\left[x,\left(X_{k}-X_{k}^{0}\right)\right]$ bei Verschiebung aus diesen Ruhelagen angibt.

Wir nennen jene Wellenfunktion der Störstelle, in der sich das Elektron im energetisch tiefsten Niveau aufhält, die Grundkonfiguration, $\psi_{\mathrm{S}}\left(x, X_{k}{ }^{0}\right)$, und zwar deshalb, weil die Wellenfunktion im Wechselspiel mit dem Gitter zustande kommt, und mit einer gewissen Ionenkonfiguration $X_{k}{ }^{0}$ im Gleichgewicht steht bei der klassischen Gittertemperatur $T=0$. Für diese Konfiguration ist nach Definition

$$
V^{(2)}\left[x,\left(X_{k}-X_{k}^{0}\right)\right] / X_{k}=X_{k}^{0}=0,
$$

und $V\left(x, X_{k}{ }^{0}\right)$ ersetzen wir in erster Näherung durch ein abgeschirmtes Coulomb-Potential der Störstelle $e^{2} / \varepsilon_{\mathrm{Ps}} r$ mit einer Abschirmungszahl $\varepsilon_{\mathrm{Ps}}$, welche gerade so gewählt ist, daß der zugehörige Energiewert einer ls-Funktion mit dem Energiewert einer stren- 
geren Rechnung zusammenfällt ${ }^{9} . \varepsilon_{\mathrm{Ps}}$ braucht daher nicht mit $\varepsilon_{\text {stat. }}$ übereinzustimmen, sondern ist eine reine Rechengröße. Gl. (34) lautet dann mit dem Ersatzpotential

$$
\begin{aligned}
& \left\{-\frac{\hbar^{2}}{2 m} \sum \frac{\partial^{2}}{\partial x^{2}}+\frac{e^{2}}{\varepsilon_{\mathrm{Ps}} r}+V^{(2)}\left[x,\left(X_{k}-X_{k}^{0}\right)\right]\right. \\
& \left.+V\left(X_{k}\right)\right\} \psi_{\mathrm{S}}=U \psi_{\mathrm{S}}
\end{aligned}
$$

und ergibt für $X_{k}=X_{k}^{0}$ die Grundkonfiguration als Lösung.

\section{§ 14. Die parameterabhängige Störstellen- wellenfunktion}

Unter der Annahme starrer Ionenhüllen hatten wir in $\S 13$ das Mehrelektronenproblem der adiabatischen Näherung auf eine Einteilchengleichung im Feld der Störstelle und der Kristallumgebung reduziert. Diese können wir dann lösen, wenn noch das Potential $V^{(2)}\left[x,\left(X_{k}-X_{k}^{0}\right)\right]$ bekannt ist. Dazu bemerken wir folgendes: im besetzten Zustand der Störstelle ergibt sich als Wechselwirkungsglied für die Gesamtheit der Gitterumgebung und der Störstelle selbst das abgeschirmte Coulomв-Potential aus (36). Führt man nun eine zusätzliche Verrückung eines Ions aus, so entsteht im Gitter ein zusätzliches Dipolmoment

$$
e e_{k} \frac{\left(X_{k}-X_{k}^{0}\right)\left(\mathfrak{r}_{k}^{0}-\mathfrak{r}\right)}{\mid \mathrm{r}_{k}^{0}-i^{3}},
$$

das nicht mehr im abgeschirmten Coulomв-Potential enthalten ist und als zusätzliches Potential auf das Elektron wirkt. Daher ist bei willkürlichen Verrückungen

$$
V^{(2)}\left[x,\left(X_{k}-X_{k}^{0}\right)\right]=\sum_{k=1}^{N} e e_{k} \frac{\left(X_{k}-X_{k}^{0}\right)\left(\mathrm{r}_{k}^{0}-\mathfrak{r}\right)}{\left|\mathrm{r}_{k}^{0}-\mathfrak{r}\right|^{3}} .
$$

Setzt man (38) in (36) ein, so kann die zugehörige Wellenfunktion durch einen Variationsansatz bestimmt werden. Wir betrachten dabei nur die Wellenfunktion des Grundzustandes. Beim Löschzentrum muß das eine s-Funktion sein, da sich das Elektron im Fall eines $Z^{++}$auf $Z$ wischengitterplatz an eine

9 Hierzu gibt es eine Anzahl von Variationsverfahren. Das einfachste, auf beliebige andere Fälle übertragbare ist die Berechnung des F-Zentren-Grundzustandes von Pekar. S. I. Pekar, Untersuchungen über die Elektronentheorie der Kristalle, Akademie-Verlag, Berlin 1954.

10 Das erste Glied in (41) ist dabei die kinetische Energie eines Elektrons mit der Wellenfunktion (39), das zweite abgeschlossene Schale anlagert, und bei einer Lücke die Konfiguration auf weitere Entfernungen wie ein wasserstoffartiges Zentrum wirkt, so daß auch hier der Grundzustand ein s-Zustand wird. Der Potentialverlauf in unmittelbarer Nähe des Zentrums hat dabei bekanntlich keinen großen Einfluß auf die Ausbildung der Wellenfunktion. (Man vgl. dazu auch die Rechnungen von Pekar über das F-Zentrum!)

Um den Sachverhalt nicht unnötig zu komplizieren, wählen wir für die Wellenfunktion der Störstelle eine 1s-Funktion

$$
\psi_{\mathrm{s}}=\pi^{-1 / 2} \alpha^{3 / 2} e^{-\alpha r}
$$

und nehmen an, daß der Charakter der Funktion bei der Verrückung nur unwesentlich verändert wird. Ihre Umgebungsabhängigkeit muß dann im Parameter $\alpha$ enthalten sein.

$$
\alpha=\alpha_{0}+\sum_{k=1}^{N} \alpha_{k}\left(X_{k}-X_{k}^{0}\right) \text {. }
$$

Einsetzen von (40) in (39), Multiplikation mit $\psi_{\mathrm{s}}{ }^{*}$ von links und Integration über $\mathfrak{r}$ liefert die Gesamtenergie als Funktion von $\alpha_{0}$ und $\alpha_{k}(k=1, \ldots, N)$, die wir abgekürzt nach (40) zusammenfassen ${ }^{10}$ in

$$
\begin{aligned}
& U\left(\alpha, X_{k}\right)=\frac{1}{2} \alpha^{2}-\frac{1}{\varepsilon_{\mathrm{Ps}}} \alpha \\
& \quad+e e_{k} \int \psi_{\mathrm{s}}^{*} \psi_{\mathrm{s}} \sum_{k=1}^{N} \frac{\left(X_{k}-X_{k}^{0}\right)\left(\mathrm{r}_{k}^{0}-\mathrm{r}\right)}{\left|\mathrm{r}_{k}^{0}-\mathfrak{r}\right|^{3}}+V\left(X_{k}\right) .
\end{aligned}
$$

Führen wir Polarkoordinaten ein und ordnen die Dipole radial auf das Zentrum gerichtet an, so zeigt sich, daß nur radiale Verschiebungen der Ionen um $\left(R_{k}-R_{k}^{0}\right)$ einen Beitrag zur Energieerhöhung liefern, dagegen nicht Verschiebungen auf der Kugelschale. Für Verschiebungen auf der Kugelschale verschwindet nämlich das skalare Produkt des Dipolmomentes mit der Summe der Aufpunktsvektoren bei kugelsymmetrischer Wellenfunktion und es bleiben nur Verschiebungen in Richtung des Radiusvektors übrig, wobei die Energieformel für $V^{(2)}$ übergeht in

$$
\int \psi_{\mathrm{s}}^{*} V^{(2)} \psi_{\mathrm{s}} \mathrm{d} \tau=e e_{k} \int \psi_{\mathrm{s}}^{*}(r) \psi_{\mathrm{s}}(r) \sum_{k=1}^{\ddot{*}} \frac{\xi_{k}}{\left|\mathfrak{r}_{k}^{0}-\mathfrak{r}\right|^{2}} \mathrm{~d} \tau,
$$

wenn wir $\left(R_{k}-R_{k}{ }^{0}\right)=\xi_{k}^{11}$ setzen. Die Konstanten

die potenielle Energie im Feld einer abgeschirmten Ladung, bei beliebigen $a$. Die Variation ist hier noch nicht durchgeführt! Vgl. P. Gombas, Theorie und Lösungsmethoden des Mehrteilchenproblems der Wellenmechanik, Verlag Birkhäuser, Basel 1950.

11 Nicht mit den Eigenschwingungen $\xi_{k}^{\mu}$ zu verwechseln! 
bestimmen sich nun durch Variation. Für die Grundkonfiguration, also $\left(R_{k}-R_{k}^{0}\right)=0$, soll $U$ in bezug auf $\alpha_{0}$ ein Minimum werden, und für beliebige $\left(R_{k}-R_{k}^{0}\right)$ muß $U$ in bezug auf die $\alpha_{k}$ ein Minimum für die richtige Wellenfunktion sein. Aus der ersten Bedingung folgt

$$
\alpha_{0}=1 / \varepsilon_{\mathrm{Ps}},
$$

und der zugehörige Energiewert ist $-\frac{1}{2} \alpha_{0}^{2}$, so daß bei Einsetzen dieses Wertes in (40) und (41) die Energieformel

$$
\begin{aligned}
U\left(\alpha_{k}, X_{k}\right)= & -\frac{1}{2} \alpha_{0}^{2}+\frac{1}{2}\left(\sum_{k} \alpha_{k} \xi_{k}\right)^{2} \\
& +\int \psi_{\mathrm{s}}^{*} V^{(2)} \psi_{\mathrm{s}} \mathrm{d} \tau+V\left(X_{k}\right)
\end{aligned}
$$

folgt. Den Wert von $\alpha_{0}$ betrachten wir im weiteren als Parameterwert und studieren die Abhängigkeit der Prozesse von seiner Größe. Die $\alpha_{k}$ lassen sich in Abhängigkeit davon eindeutig bestimmen. Entwickelt man nämlich im Integral die Wellenfunktion nach Potenzen von $\xi_{k}$ und ersetzt

$$
\text { so entsteht } \begin{aligned}
U\left(\alpha_{k} X_{k}\right)=-\frac{1}{2} \alpha_{0}^{2}+\frac{1}{2}\left(\sum_{k} \alpha_{k} \xi_{k}\right)^{2} & +e e_{j} \int \sum_{j} \psi_{\mathrm{s} 0}^{*} \psi_{\mathrm{s} 0} \frac{\xi_{j}}{\left|\mathrm{r}_{j}^{0}-\mathfrak{r}\right|^{2}} \mathrm{~d} \tau \\
& +\sum_{k} 2 \alpha_{k} \xi_{k} \int \sum_{j} \psi_{\mathrm{s} 0}^{*} \frac{\partial \psi_{\mathrm{s} 0}}{\partial \alpha} \frac{\xi_{j}}{\left|\mathfrak{r}_{j}^{0}-\mathfrak{r}\right|^{2}} \mathrm{~d} \tau+\ldots+V\left(X_{k}\right),
\end{aligned}
$$$$
\frac{\partial \psi_{\mathrm{s}}}{\partial \xi_{k}} \quad \text { durch } \frac{\partial \psi_{\mathrm{s}}}{\partial \alpha} \frac{\partial \alpha}{\partial \xi_{k}}=\frac{\partial \psi_{\mathrm{s}}}{\partial \alpha} \alpha_{k},
$$

wobei mit $\psi_{\mathrm{s} 0}$ die Einfangwellenfunktion bezeichnet wird, d. h. jene, die an der Stelle $\alpha_{0}$ genommen wird. Die Energie soll bei beliebigem $\xi_{k}$ ein Minimum in bezug auf die $\alpha_{i}$ werden. Daraus folgt bei Variation unter Beachtung der Unabhängigkeit der $\xi_{k}$

$$
\alpha_{i}=-2 \int \psi_{\mathrm{s} 0}^{*} \frac{\partial \psi_{\mathrm{s} 0}}{\partial \alpha} \frac{1}{\left|\mathrm{r}_{i}^{0}-\mathfrak{r}\right|^{2}} \mathrm{~d} \tau,
$$

wenn man höhere als lineare Glieder vernachlässigt. Nun ist aber wegen der Eigenschwingungsdarstellung (31)

$e e_{k} \int \psi_{\mathrm{s} 0}^{*} \frac{\partial \psi_{\mathrm{s} 0}}{\partial \alpha} \frac{1}{\left|\mathfrak{r}_{k}^{0}-\mathfrak{r}\right|^{2}} \mathrm{~d} \tau=\frac{1}{2} \frac{\partial}{\partial \alpha} \xi_{k}^{1} \gamma^{1}$,

wenn die Eigenschwingung als Funktion des Parameters $\alpha$ der 1s-Funktion aufgefaßt wird. Entwickelt man in (40) $\left(R_{k}-R_{k}{ }^{0}\right)$ nach den Eigenschwingungen des Grundzustandes, so wird

$$
\left(R_{k}-R_{k}^{0}\right)=\sum_{\mu} \xi_{k}^{\mu} q_{\mu}^{\mathrm{s}} .
$$

Beachtet man weiter die aus den Orthogonalitätseigenschaften der Eigenvektoren folgenden Relationen

$$
\sum_{k}\left(\frac{\partial}{\partial \alpha} \xi_{k}^{1} \gamma^{1}\right) \xi_{k}^{1}=\frac{\partial}{\partial \alpha} \gamma^{1}
$$

und $\sum_{k}\left(\frac{\partial}{\partial \alpha} \xi_{k}^{1} \gamma^{1}\right) \xi_{k}^{\mu}=-\sum_{k} \xi_{k}^{1} \gamma^{1}\left(\frac{\partial}{\partial \alpha} \xi_{k}^{\mu}\right)=0$ (wenn man annimmt, daß die übrigen zu $\xi_{k}{ }^{1}$ orthogonalen Eigenschwingungen nicht wesentlich von $\alpha$ abhängig sind), dann wird mit (49), (50) und (51)

$$
\sum_{k} \alpha_{k}\left(R_{k}-R_{k}^{0}\right)=-2\left(\frac{\partial}{\partial \alpha} \gamma^{1}\right) q_{1}^{\mathrm{s}}
$$

und die Wellenfunktion lautet

$\psi_{\mathrm{s}}=\pi^{-1 / 2}\left(\alpha_{0}-2 \frac{\partial \gamma^{1}}{\partial \alpha} q_{1}^{\mathrm{s}}\right)^{3 / 2} \exp \left[-\left(\alpha_{0}-2 \frac{\partial \gamma}{\partial \alpha} q_{1}^{\mathrm{s}}\right) r\right]$.

In gleicher Weise können mit einem Variationsansatz für die Parameter die Wellenfunktionen angeregter Zustände des Störstellenelektrons bestimmt werden, wenn man sie der zusätzlichen Bedingung der Orthogonalität zum Grundzustand unterwirft. Diese Wellenfunktionen müssen für die Berechnung der Übergangswahrscheinlichkeiten bei inneren Anregungen und der nachfolgenden Ablösung des Elektrons in das Leitfähigkeitsband bekannt sein. Ein Beispiel dafür bieten die Reaktionen des F-Zentrums. Für den Einfang aus dem Leitfähigkeitsband dagegen ist der Grundzustand weitaus der maßgebende. Da die Reaktionen des F-Zentrums in einer gesonderten Arbeit dargestellt werden sollen, beschränken wir uns auf die Einfangreaktionen und demnach die im vorangehenden abgeleitete Funktion des Grundzustandes. 


\section{§ 15. Gitterenergie beim Einfang}

Von jenen für die Berechnung der Übergangswahrscheinlichkeiten notwendigen Größen fehlen uns nach den vorangegangenen Untersuchungen nur noch zwei: Die Nullpunktsverschiebung der Störstellenschwingung und ihre Frequenzänderung. Wegen der Annahme verschwindender Dispersion und der in $\S 12$ durchgeführten Betrachtungen vernachlässigen wir die Frequenzänderungen, die durch Einfang oder Ionisation eines Elektrons in der Störstelle entstehen. Die Eigenschwingungen $\xi_{i}{ }^{\mu \mathrm{S}}, \xi_{i}{ }^{\mu \mathrm{L}}, \xi_{i}{ }^{\mu \mathrm{V}}$, die den verschiedenen Quantenzuständen des Störstellenelektrons entsprechen (siehe $\S 16$ ), sind daher in dieser Näherung gleich. In derselben Näherung können die Verschiebungen des Gitters durch eine einzige Eigenschwingung ausgedrückt werden. Dies bedeutet, daß bei einer Fourier-Zerlegung der Verschiebung nach den Normalkoordinaten nur eine einzige Komponente in bezug auf die eben erwähnte Eigenschwingung $\xi_{i}{ }^{1 \mathrm{~L}, \mathrm{~S}, \mathrm{~V}}$ auftritt und sich die Transformation (12) bzw. (13) auf ${ }^{12}$

$$
q_{1}^{\mathrm{s}}=q_{1}^{\mathrm{L}}+a_{1}^{(\mathrm{L}, \mathrm{S})}, \quad q_{j}^{\mathrm{s}}=q_{j}^{\mathrm{L}}
$$

vereinfacht, wenn man einen Übergang zwischen dem Leitungsband und der Störstelle betrachtet.

Die Konstante $a_{1}{ }^{(\mathrm{L}, \mathrm{S})}$ gewinnt man nun aus einer Minimalforderung an die Gesamtenergie.

Nach (46) wird bei Einsetzen der durch die Variation berechneten Werte und Vernachlässigung von Termen, die zu einer Frequenzänderung führen würden,

$$
U_{\mathrm{s}}\left(X_{k}\right)=-\frac{1}{2} \alpha_{0}^{2}+\gamma^{1} q_{1}^{\mathrm{s}}+V\left(q_{1}^{\mathrm{s}} \ldots q_{N}^{\mathrm{s}}\right),
$$

wobei $V\left(q_{1}^{\mathbf{s}} \ldots q_{N}^{\mathbf{s}}\right)$ die Transformierte von $V\left(X_{k}\right)$ ist.

Da diese Energie für $q_{j}^{\mathrm{s}}=0$ ein Minimum haben soll, so müssen die linearen Glieder verschwinden. Das bedeutet, daß $V$ die Gestalt

$$
V\left(q_{1}^{\mathrm{s}} \ldots q_{N}^{\mathrm{s}}\right)=-\gamma^{1} q_{1}^{\mathrm{s}}+\frac{1}{2} M \omega^{2} \sum_{j=1}^{N} q_{j}^{\mathrm{s} 2}
$$

haben muß.

Geht das Elektron in das Leitungsband über, so kann man wegen der weit ausgebreiteten Wellenfunktion eine Wechselwirkung mit dem Gitter vernachlässigen und erhält

$$
U_{\mathrm{L}}\left(X_{k}\right) \approx E_{\mathrm{L}}+V=E_{\mathrm{L}}-\gamma^{1} q_{1}^{\mathrm{s}}+\frac{1}{2} M \omega^{2} \sum_{j=1}^{N} q_{j}^{\mathrm{s} 2} .
$$

12 Über die Bedeutung der auftretenden Koeffizienten siehe $\S 10$ in H. Stumpf, Z. Naturforschg. 10 a, 971 [1955].
In der Normalkoordinatenstellung muß

$$
\frac{\partial U_{\mathrm{L}}}{\partial q_{i}^{\mathrm{L}}}=0 \quad \text { sein für } \quad q_{i}^{\mathrm{L}}=0 \text {. }
$$

Dies ist nach Definition die Ruhelage der $q_{i}^{\mathrm{L}}$. Daraus folgt mit (54)

$$
a_{1}^{(\mathrm{L}, \mathrm{S})}=\frac{\gamma^{1}}{M \omega^{2}}
$$

und die statische Gitterenergie des Einfangs wird

$$
V\left(q_{1}^{\mathrm{L}} \ldots q_{N}^{\mathrm{L}}\right)-V\left(q_{1}^{\mathrm{L}}+a_{1}^{(\mathrm{L}, \mathrm{S})}, \ldots, q_{N}^{\mathrm{L}}\right)=\frac{1}{2} M \omega^{2} a_{1}^{(\mathrm{L}, \mathrm{S}) 2} .
$$

Dasselbe Ergebnis hätte man mit Hilfe der Rechnungen in $\S 12$ ableiten können. Wir haben jedoch diesen Weg gewählt, um die Operationen an dem quantenmechanischen Energieausdruck vornehmen zu können.

\section{§ 16. Löschzentren Einfangs- und Ionisations- matrizen}

Mit Hilfe der Untersuchungen in den vorangehenden Paragraphen lassen sich nun die Übergangsmatrizen berechnen. Für die Elektronenübergänge am Löschzentrum verwenden wir dabei drei Typen von Gesamtwellenfunktionen:

1. Das Störelektron befinde sich im Leitungsband, in einer sonst beliebigen elektronischen Umgebung, die durch die Anregungsverhältnisse definiert ist. Die zugehörige Wellenfunktion sei

$$
\psi_{\mathrm{L}}\left(x, X_{k}\right) \psi_{n}\left(x_{i} X_{k}\right) \Phi_{l_{1}^{\mathrm{L}}}^{\mathrm{L}} \ldots \Phi_{l_{N}^{\mathrm{L}}}^{\mathrm{L}} .
$$

2. Das Störstellenelektron sei im Löschzentrum und der Quantenzustand $n$ der Umgebung bleibe unverändert. Die Wellenfunktion lautet dann

$$
\psi_{\mathrm{S}}\left(x, X_{k}\right) \psi_{n}\left(x_{i} X_{k}\right) \Phi_{l_{1}^{\mathrm{S}}}^{\mathrm{S}} \ldots \Phi_{l_{N}^{\mathrm{S}}}^{\mathrm{S}} .
$$

3. Bei immer noch unveränderter Umgebung sei das Störelektron im Valenzband

$$
\psi_{\mathrm{V}}\left(x, X_{k}\right) \psi_{n}\left(x_{i} X_{k}\right) \Phi_{l_{1}^{\mathrm{V}}}^{\mathrm{V}} \ldots \Phi_{l_{N}^{\mathrm{V}}}^{\mathrm{V}} .
$$

Die zugehörigen Kernwellenfunktionen können dabei die gesamte Variabilität ihrer Anregungen ausnutzen, ebenso denken wir uns die $\psi_{n}$ nur dadurch beschränkt, daß sie physikalisch realisierbare Verhältnisse angeben sollen, d. h. gegenüber der Elektronenzahl im Grundzustand des Valenzbandes nur eine geringe Anzahl angeregter Elektronen im Leitungsband enthalten sollen. 
Wir beginnen nun mit den Übergangselementen für Ionisation des Störzentrums durch kristalleigene Störglieder. Verzichtet man in erster Näherung auf alle Mehrteilchenoperatoren, so bleibt nach I, § 9

$$
\begin{aligned}
& -\frac{\hbar^{2}}{M} \sum_{k} \int \psi_{\mathrm{L}}^{*} \frac{\partial}{\partial X_{k}} \psi_{\mathrm{S}} \Phi_{l_{1}^{\mathrm{L}}}^{\mathrm{L}} \ldots \Phi_{l_{N}^{*}}^{\mathrm{L}} \partial X_{k}\left[\Phi_{l_{1}^{\mathrm{S}}}^{\mathrm{S}} \ldots \Phi_{l_{N}^{\mathrm{S}}}^{\mathrm{S}}\right] \mathrm{d} \tau \\
& -\frac{\hbar^{2}}{2 M} \frac{\mathrm{S}}{k} \int \psi_{\mathrm{L}}^{*} \frac{\partial^{2}}{\partial X_{k}^{2}} \psi_{\mathrm{S}} \Phi_{l_{1}^{\mathrm{L}}}^{\mathrm{L} *} \ldots \Phi_{l_{N}^{\mathrm{L}}}^{\mathrm{L}} \Phi_{l_{1}^{\mathrm{S}}}^{\mathrm{S}} \ldots \Phi_{l_{N}^{\mathrm{s}}}^{\mathrm{S}} \mathrm{d} \tau,(63)
\end{aligned}
$$

und bei Einführung von Normalkoordinaten entsteht dann, da $\psi_{\mathrm{S}}$ nur von $q_{1}^{\mathrm{S}}$ abhängig ist ${ }^{13}$

$$
\begin{aligned}
& -\frac{\hbar^{2}}{M} \int \psi_{\mathrm{L}}^{*} \frac{\partial}{\partial q_{1}^{\mathrm{S}}} \psi_{\mathrm{s}} \Phi_{l_{1}^{\mathrm{L}}}^{\mathrm{L}}\left(q_{1}^{\mathrm{L}}\right) \frac{\partial}{\partial q_{1}^{\mathrm{S}}} \Phi_{l_{1}^{\mathrm{s}}}^{\mathrm{S}}\left(q_{1}^{\mathrm{S}}\right) \mathrm{d} \tau \\
& -\frac{\hbar^{2}}{2 M} \int \psi_{\mathrm{L}}^{*} \frac{\partial^{2}}{\partial q_{1}^{\mathrm{S} 2}} \psi_{\mathrm{S}} \Phi_{l_{1}^{\mathrm{L}}}^{\mathrm{L}}\left(q_{1}^{\mathrm{L}}\right) \Phi_{l_{1}^{\mathrm{S}}}^{\mathrm{S}}\left(q_{1}^{\mathrm{S}}\right) \mathrm{d} \tau .
\end{aligned}
$$

Dieses Matrixelement setzt die Kenntnis von $\psi_{\mathrm{L}}$ voraus. In erster Näherung setzen wir

$$
\psi_{\mathrm{L}}=V^{-1 / 2},
$$

wobei $V$ das in $\S 11$ definierte Grundvolumen bedeutet. Bei einer strengeren Rechnung müßte der Einfluß der Störstelle durch die Beimengung einer wasserstoffartigen Lösung berücksichtigt werden. Jedoch können wir wegen der starken Abschirmung der Störstelle und dem verhältnismäßig kleinen Grundvolumen $V$ eine annähernd homogene Verteilung der Dichte voraussetzen, ohne schwerwiegende Fehler zu begehen. Entwickelt man $\psi_{\mathrm{S}}$ in eine TAYLOR-Reihe an der Stelle $q_{1}^{\mathrm{S}}=0$

$$
\begin{aligned}
\psi_{\mathrm{S}}\left(x, q_{1}^{\mathrm{S}}\right)=\psi_{\mathrm{S}}(x, 0) & +\frac{\partial}{\partial q_{1}^{\mathrm{S}}} \psi_{\mathrm{S} / q_{1}^{\mathrm{S}}=0} q_{1}^{\mathrm{S}} \\
& +\frac{1}{2} \frac{\partial^{2}}{\partial q_{1}^{\mathrm{S} 2}} \psi_{\mathrm{S} / q_{1}^{\mathrm{S}}=0} q_{1}^{\mathrm{S} 2}+\ldots,
\end{aligned}
$$

so wird das Integral

$$
\int \psi_{\mathrm{L}}^{*} \frac{\partial}{\partial q_{1}^{\mathrm{s}}} \psi_{\mathrm{S}} \mathrm{d} x
$$

wenn man in ihm nach den Gliedern nullter Ordnung der eingesetzten TAYLOR-Reihe abbricht, gleich

$$
\int \psi_{\mathrm{L}}^{*} \frac{\partial}{\partial q_{1}^{\mathrm{s}}} \psi_{\mathrm{s} / q_{\mathrm{1}}^{\mathrm{S}}=0} \mathrm{~d} x=-36 \pi^{1 / 2} V^{-1 / 2} C \alpha_{0}^{-5 / 2},
$$

wobei die Konstante

$$
C=-2 \frac{\partial \gamma^{1}}{\partial \alpha}
$$

ist. In derselben Weise ergibt das zweite Integral

$$
\int \psi_{\mathrm{L}}^{*} \frac{\partial^{2}}{\partial q_{1}^{\mathrm{S} 2}} \psi_{\mathrm{s} / q_{1}^{\mathrm{S}}=0} \mathrm{~d} x=-198 \pi^{1 / 2} V^{-1 / 2} C^{2} \alpha_{0}^{-7 / 2} \text {. }
$$

Damit erhält man, unter Anwendung von (9) und (15) auf das erste Glied in (64)

und

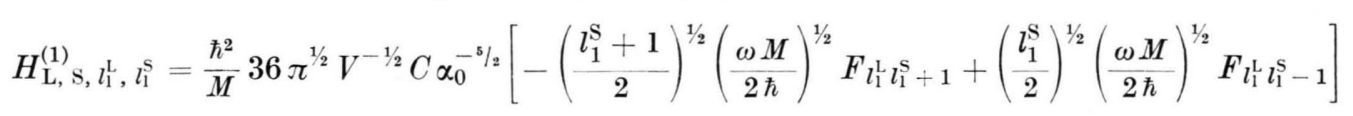

$$
H_{\mathrm{L}, \mathrm{S}, l_{1}^{\mathrm{L}}, l_{1}^{\mathrm{S}}}^{(2)}=\frac{\hbar^{2}}{2 M} 198 \pi^{1 / 2} V^{-1 / 2} C^{2} \alpha_{0}^{-7 / 2} F_{l_{1}^{\mathrm{L}} l_{1}^{\mathrm{S}}},
$$

bzw. die entsprechenden Ionisationsmatrixelemente für die Ionisation der Störstelle bei Übergang des Elektrons in das Valenzband, wenn auch für die Wellenfunktion am oberen Rand des Valenzbandes in erster Näherung (65) gesetzt wird. Damit sind die strahlungslosen Ionisationsmatrizen für Übergänge von Elektronen in das Leitungs- bzw. Valenzband berechnet.

Wir gehen nun zu jenen Matrizen über, die den Einfang eines Elektrons aus dem Valenz- oder Leitungsband in die Störstelle beschreiben. Da nicht bekannt ist, ob die nichtadiabatischen Störglieder hermitesche Operatoren sind, kann man die Einfangmatrizen nicht durch Übergang zu den adjungierten Ionisationsmatrizen erhalten, sondern muß sie explizit berechnen bzw. ihre Hermitezität nachweisen.

Wir betrachten dazu das erste Glied von (63), aber sogleich auf Normalkoordinaten transformiert.

Dieses lautet $\quad-\frac{\hbar^{2}}{M} \sum_{k} \int \psi_{\mathrm{S}}^{*}\left[\frac{\partial}{\partial q_{k}^{\mathrm{S}}} \psi_{\mathrm{L}}\right] \Phi_{l_{1}^{\mathrm{S}}}^{\mathrm{S}} \ldots \Phi_{l_{N}^{\mathrm{S}}}^{\mathrm{S}} \Phi_{l_{1}^{\mathrm{L}}}^{\mathrm{L}} \ldots\left[\frac{\partial}{\partial q_{k}^{\mathrm{S}}}\right] \Phi_{l_{K}^{\mathrm{L}}}^{\mathrm{L}} \ldots \Phi_{l_{N}^{\mathrm{L}}}^{\mathrm{L}} \mathrm{d} \tau$

13 Die übrigen Normalschwingungen bleiben unverändert und werden im folgenden gar nicht mehr in der Indizie- rung der Matrixelemente und in den Elementen selbst angeführt, siehe (I, 53, 54). 


\begin{tabular}{|c|c|c|c|c|c|c|}
\hline $\begin{array}{l}\text { berechnet bei } \\
\text { unter folgenden } \\
\text { Vorraussetzungen }\end{array}$ & Huang-Rhys ${ }^{14}$ & $\begin{array}{l}\text { Pekar }^{15} \\
\text { TrlifaJ }\end{array}$ & TEWORDT ${ }^{16}$ & Meyer $^{17}$ & HeLmis ${ }^{18}$ & StumpF \\
\hline Gitter & $\begin{array}{l}\text { quantisiertes } \\
\text { Kontinuum }\end{array}$ & $\begin{array}{l}\text { quantisiertes } \\
\text { Kontinuum }\end{array}$ & atomistisch & atomistisch & atomistisch & atomistisch \\
\hline $\begin{array}{l}\text { Elektronen- } \\
\text { polarisation }\end{array}$ & phänomenol. & phänomenol. & $\begin{array}{l}\text { atom. (nicht aus- } \\
\text { geführt) }\end{array}$ & phänomenol. & - & $\begin{array}{l}\text { atomistisch } \\
\text { (in Teil V) }\end{array}$ \\
\hline $\begin{array}{l}\text { Elektronenwel- } \\
\text { lenfunktionen }\end{array}$ & $\begin{array}{l}\text { Einelektronen- } \\
\text { funktionen }\end{array}$ & $\begin{array}{l}\text { Einelektronen- } \\
\text { funktionen }\end{array}$ & $\begin{array}{l}\text { Gesamtwellen- } \\
\text { funktionen }\end{array}$ & $\begin{array}{l}\text { Einelektronen- } \\
\text { funktionen }\end{array}$ & $\begin{array}{l}\text { Einelektronen- } \\
\text { funktionen }\end{array}$ & $\begin{array}{l}\text { Gesamtwellen- } \\
\text { funktionen }\end{array}$ \\
\hline Gitterübergänge & $\begin{array}{l}\text { einquanten beim } \\
\text { einzeln. Oszillator } \\
V \rightarrow \infty\end{array}$ & $\begin{array}{l}\text { einquanten beim } \\
\text { einzeln. Oszillator } \\
V \rightarrow \infty\end{array}$ & $\begin{array}{l}\text { einquanten beim } \\
\text { einzeln. Oszillator } \\
V \rightarrow \infty\end{array}$ & $\begin{array}{l}\text { einquanten beim } \\
\text { einzeln. Oszillator } \\
V \rightarrow \infty\end{array}$ & $\begin{array}{l}\text { einquanten I. Ord. } \\
V \rightarrow \infty\end{array}$ & $\begin{array}{l}\text { mehrquanten } \\
V \text { endlich }\end{array}$ \\
\hline $\begin{array}{l}\text { Frequenzspek- } \\
\text { trum }\end{array}$ & $\begin{array}{l}\text { optisch, eine } \\
\text { Frequenz }\end{array}$ & $\begin{array}{l}\text { optisch, eine } \\
\text { Frequenz }\end{array}$ & $\begin{array}{l}\text { akustisch } \\
\text { mehr. Frequ. }\end{array}$ & $\begin{array}{l}\text { optisch, eine } \\
\text { Frequenz }\end{array}$ & $\begin{array}{l}\text { optisch, mehr. } \\
\text { Frequenzen }\end{array}$ & $\begin{array}{l}\text { optisch, eine } \\
\text { Frequenz }\end{array}$ \\
\hline $\begin{array}{l}\text { Frequenzände- } \\
\text { rungen }\end{array}$ & keine & keine & keine & keine & ja & keine \\
\hline $\begin{array}{l}\text { Thermisches } \\
\text { Gleichgewicht }\end{array}$ & ja & ja & ja & ja & ja & nein \\
\hline $\begin{array}{l}\text { Störungsrech- } \\
\text { nung I. Ordn. }\end{array}$ & ja & ja & ja & ja & höherer Ordn. & ja \\
\hline $\begin{array}{l}\text { FRANCK-Condon- } \\
\text { Näherung }\end{array}$ & ja & ja & ja & nein & nein & ja \\
\hline $\begin{array}{l}\text { Störstellen- } \\
\text { schwingung }\end{array}$ & nein & nein & nein & nein & nein & ja \\
\hline $\begin{array}{l}\text { fixierte Stör- } \\
\text { niveaus }\end{array}$ & ja & ja & nein & ja & - & nein \\
\hline Numerische Werte & $\begin{array}{l}\text { direkt berechn. } \\
\text { für F-Zentren }\end{array}$ & $\begin{array}{l}\text { direkt berechn. } \\
\text { für F-Zentren }\end{array}$ & $\begin{array}{l}\text { größenordn.- } \\
\text { mäßige Abschät- } \\
\text { zungen in homöo- } \\
\text { pol. Kristallen }\end{array}$ & $\begin{array}{l}\text { berechn. für } \\
\text { F-Zentren }\end{array}$ & - & $\begin{array}{l}\text { direkt berechn. } \\
\text { für beliebige Stö- } \\
\text { rungen in pol. } \\
\text { Kristallen mit } \\
\text { s-Grundzustands- } \\
\text { Funktion }\end{array}$ \\
\hline
\end{tabular}

Tab. 1. Übergangswahrscheinlichkeiten.

14 K. Huang u. A. Rhys, Proc. Roy. Soc., Lond. A 204, 406

15 Siehe Anm. 9. - M. TrlifaJ, Czechosl. J. Phys. 5, 133 [1955].
16 L. Tewordt, Z. Phys. 137, 604 [1954].

17 H. J. G. Meyer, Halbleiterprobleme III, Verlag Vieweg, Braunschweig 1956, S. 230.

18 G. Helmis, Ann. Phys., Lpz. 17, 356 [1957] ; Ann. Phys., Lpz. 19, 41 [1957]. 
und durch Anwendung der Formel (9) geht (71) über in

$-\hbar^{2} \sum_{k} \int \psi_{\mathrm{S}}^{*}\left[\frac{\partial}{\partial q_{k}^{\mathrm{S}}} \psi_{\mathrm{L}}\right] \Phi_{l_{1}^{\mathrm{S}} \ldots}^{\mathrm{S}} \ldots \Phi_{l_{N}^{\mathrm{S}}}^{\mathrm{S}} \Phi_{l_{1}^{\mathrm{L}}}^{\mathrm{L}} \ldots\left[-\left(\frac{l_{k}^{\mathrm{L}}+1}{2}\right)^{1 / 2}\left(\frac{\omega M}{2 \hbar}\right)^{1 / 2} \Phi_{l_{K}^{\mathrm{L}}-1}^{\mathrm{L}}+\left(\frac{l_{k}^{\mathrm{L}}}{2}\right)^{1 / 2}\left(\frac{\omega M}{2 \hbar}\right)^{1 / 2} \Phi_{l_{K}^{\mathrm{L}}+1}^{\mathrm{L}}\right] \ldots \Phi_{l_{N}^{\mathrm{L}}}^{\mathrm{L}} \mathrm{d} \tau$.

Jetzt ist die Gestalt von (69) erreicht. Da aber die $q$-Abhängigkeit der Leitungs- bzw. Valenzbandfunktionen nicht bekannt ist, müssen wir den Ausdruck durch partielle Integration solange umformen, bis die Differentiation nach den Normalkoordinaten auf $\psi_{\mathrm{S}}$ übergegangen ist. Man erhält zunächst

$$
\frac{\hbar^{2}}{M} \sum_{k} \int \psi_{\mathrm{L}} \frac{\partial}{\partial q_{k}^{\mathrm{S}}}\left\{\psi_{\mathrm{S}}^{*} \Phi_{l_{1}^{\mathrm{S}}}^{\mathrm{S}} \ldots \Phi_{l_{N}^{\mathrm{S}}}^{\mathrm{S}} \Phi_{l_{1}^{\mathrm{L}}}^{\mathrm{L}} \ldots\left[-\left(\frac{l_{k}^{\mathrm{L}}+1}{2}\right)^{1 / 2}\left(\frac{\omega M}{2 \hbar}\right)^{1 / 2} \Phi_{l_{\mathrm{K}}-1}^{\mathrm{L}}+\left(\frac{l_{k}^{\mathrm{L}}}{2}\right)^{1 / 2}\left(\frac{\omega M}{2 \hbar}\right)^{1 / 2} \Phi_{l_{K}^{\mathrm{L}}+1}^{\mathrm{L}}\right] \ldots \Phi_{l_{N}^{\mathrm{L}}}^{\mathrm{L}}\right\} \mathrm{d} \tau,
$$

wenn man beachtet, daß wegen der Endlichkeit der Elektronenfunktion und dem exponentiellen Abklingen der Kernfunktionen im Unendlichen des $q$-Raumes die übrigen Glieder verschwinden. Führt man nun die Differentiation an den einzelnen Funktionen in (73) aus und beachtet, daß streng (auch wenn die angenommenen Näherungsfunktionen dies nicht vollständig erfüllen) gelten muß

$$
\int \psi_{\mathrm{L}}(x, q) \psi_{\mathrm{S}}^{*}(x, q) \mathrm{d} x=0
$$

so reduziert sich das Integral (73) auf

$$
\frac{\hbar^{2}}{M} \int \psi_{\mathrm{L}} \frac{\partial}{\partial q_{1}^{\mathrm{S}}} \psi_{\mathrm{S}}^{*} \Phi_{l_{1}^{\mathrm{S}}}^{\mathrm{S}}\left[-\left(\frac{l_{1}^{2}+1}{2}\right)^{1 / 2}\left(\frac{\omega M}{2 \hbar}\right)^{1 / 2} \Phi_{l_{1}^{\mathrm{L}}-1}^{\mathrm{L}}+\left(\frac{l_{1}^{\mathrm{L}}}{2}\right)^{1 / 2}\left(\frac{\omega M}{2 \hbar}\right)^{1 / 2} \Phi_{l_{1}^{\mathrm{L}}+1}^{\mathrm{L}}\right] \mathrm{d} x \mathrm{~d} q_{1}^{\mathrm{S}},
$$

und wegen der Realität der verwendeten Funktionen folgt daraus

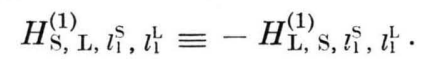

Eine weitere Untersuchung mit Hilfe der Symmetrieeigenschaften von $F_{l}^{\mathrm{s}} l_{1}^{\mathrm{L}}$ und dem Ziel die Hermitezität nachzuweisen, stellen wir hier nicht an, da bereits (76) explizit nach (69) berechnet werden kann.

Unter den einschränkenden Voraussetzungen (66) kann man ebenso leicht nachweisen, daß

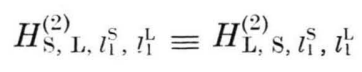

gelten muß. Auch hier versuchen wir nicht die vollständige Hermitezität nachzuweisen, sondern begnügen uns mit der Berechenbarkeit der Einfangmatrizen (76) und (77) nach (69).

In genau derselben Weise kann man die Valenzband-Einfangmatrizen berechnen.

Wir bemerken noch, daß mit den eben entwickelten Hilfsmitteln auch die inneren Übergänge eines Störzentrums beschrieben werden können, wenn man an Stelle von $\psi_{\mathrm{L}}$ einen gebundenen Anregungszustand $\psi_{\mathrm{SA}}$ der Störstelle einsetzt.

Die thermischen Übergangsmatrizen am Löschzentrum lassen sich hiermit vollständig berechnen.
Man sieht sofort, daß die optischen Übergangsmatrizen bei der Wahl von (65) und (53) verschwinden müssen. Ein Zentrum, das als Grundzustand einen s-Zustand besitzt, muß daher vornehmlich als Löschzentrum wirken. Diese Tatsache können wir jedoch noch nicht für eine systematische Klassifizierung der Störzentren unmittelbar verwenden, da die Näherung (65) in kubischen Gittern nur ganz in der Nähe des unteren Leitungs- bzw. oberen Valenzbandrandes in ihren Symmetrieeigenschaften (auf die es bei den optischen Dipolmatrizen entscheidend ankommt) mit der strengen Lösung übereinstimmen kann, und die thermischen Übergänge noch von den Energieverhältnissen, wie sich später zeigen wird, abhängen, so daß also strahlende Quadrupole z. B. eine ernsthafte Konkurrenz bilden könnten. Ferner wurden Rekombinationen mit angeregten Zuständen ausgeschlossen. All diese feineren Einzelheiten müssen einer genauen Untersuchung unterworfen werden, bevor unser vorläufig definiertes Löschzentrum als charakteristisch für ein Löschzentrum im physikalischen Sinne angesehen werden darf.

Die zu den Matrixelementen (69) (70) gehörigen Übergangswahrscheinlichkeiten und ihre numerischen Werte folgen in Teil IV. 